\title{
A Unified Analytical Look at Reynolds Flocking Rules
}

\author{
Reza Olfati Saber \\ Control and Dynamical Systems \\ California Institute of Technology \\ Pasadena, CA 91125 \\ e-mail: olfati@cds.caltech.edu
}

September 4, 2003

Submitted to the American Control Conference 2004, Boston, MA

\begin{abstract}
In this paper, we present a unified theoretical view of the so-called "Flocking Rules of Reynolds" introduced in 1987. No equations describing the rules or mathematical models of the mobile agents known as "boids" were presented in the original work by Reynolds. We show how to model a group of autonomous mobile agents by dynamic nets and achieve flocking by dissipation of the structural energy of the multi-agent system. As a by-product, we obtain a single protocol called the $(\alpha, \alpha)$ protocol that encompasses all three flocking rules of Reynolds. We provide geometric interpretations of the advanced forms of some of these flocking rules. Simulation results are provided that demonstrate flocking of 100 agents towards a sink.
\end{abstract}

\section{Introduction}

Flocking is a group behavior of large number of agents with a common objective. In English it is described as "moving together in large numbers". In [11], Reynolds introduced three flocking rules in English that are quoted in the following (after a change in order)

1) "Flock Centering: attempt to stay close to nearby flockmates,"

2) "Obstacle Avoidance: avoid collisions with nearby flockmates,"

3) "Velocity Matching: attempt to match velocity with nearby flockmates."

These rules describe the behavior of each agent in interaction with other neighboring agents (or flockmates). The third rule is also known as the "Alignment" rule if read as "attempt to match attitude with nearby flockmates". The problem is that expressions like "attempt to stay close" and "attempt to match velocity" have broad meanings and are open to various interpretations.

The primary reason that it is worth to look deeper for a more specific and analytical form of these rules is that they in fact led to creation of the first animation of flocking. This animation 
closely resembles flocking by birds. One approach is to express all or some of the three rules mathematically and then analyze the resulting multi-agent system. This approach is taken by Vicsek et al. in [12] only for simulation of the "Alignment" rule. A similar approach is pursued by Helbing et al. [3] for the "escape panic" phenomenon without ever mentioning any connections to flocking. In the escape panic phenomenon, large number of people flock out of a crowded area with very few exits. Thus, "escape panic" can be viewed as a special case of flocking. The common objective of agents in escape panic is to leave a room/building as soon as possible.

Our approach to flocking is fundamentally different and does not rely on direct mathematical translation of the Reynolds rules. In [6], we partially base our theory on the following principle: "the primary objective of each agent in a flock is to maintain a fixed inter-agent distance with all of its neighboring agents." We refer to each member of a flock as an $\alpha$-agent and this principle is called the task of an $\alpha$-agent. In [6, 10], a theoretical framework is developed for flocking in presence of multiple obstacles. In particular, a single protocol called the $(\alpha, \alpha)$ protocol is provided that led to creation of flocking.

The main contribution of this paper is to demonstrate that the $(\alpha, \alpha)$ protocol encompasses all three rules of Reynolds as special cases. The $(\alpha, \alpha)$ protocol is stated explicitly in a single equation. We decompose the terms of the $(\alpha, \alpha)$ protocol in two categories, namely, motion planning terms and velocity matching terms. After stating both type of terms in English, we conclude that the motion planning terms encompasses the first two rules of Reynolds and the velocity matching terms are equivalent to the third rule.

Some of the past research related to flocking/swarming can be summarized as follows. An attitude alignment problem similar to the one in Viscek et al. [12] was recently investigated by Jadbabaie et al. [4] and a convergence analysis was provided for asymptotic alignment. The use of gyroscopic forces with connections to flocking is also considered in Chang et al. [1]. To the best of our knowledge, the analysis of the other two flocking rules with a motion planning nature has never been presented in the literature. In Liu et al. [5], stability analysis of swarms with fixed interconnection topology is studied. Furthermore, Gazi and Passino [2] use social potentials to create cohesion in swarms.

An outline of the paper is as follows: in Section 2 basic notions in a dynamic graph theoretic framework of flocking are presented. In Section 3, velocity matching or alignment is discussed as a velocity consensus problem. In Section 4, our main results on the relation between the $(\alpha, \alpha)$ protocol and Reynolds rules are presented. Simulation results for flocking of 100 agents are given in Section 5. Finally, in Section 6, concluding remarks are made.

\section{Basic Notions: Nets, Flocks, and Dynamic Nets}

In this section, we define some basic notions of a theoretical framework for flocking that is presented in $[10,6]$. Consider a set of dynamic agents with dynamics

$$
\left\{\begin{array}{c}
\dot{q}_{i}=p_{i} \\
\dot{p}_{i}=u_{i}
\end{array}\right.
$$

where $q_{i}, p_{i}, u_{i} \in \mathbb{R}^{d}$ (e.g. $d=2,3$ ) denote the position, velocity, and control input of the $i$ th agent, respectively, with $i \in \mathcal{I}=\{1, \ldots, n\}$. Let $B_{i}=B\left(q_{i}, r\right)$ denote a closed ball with radius $r>0$ 


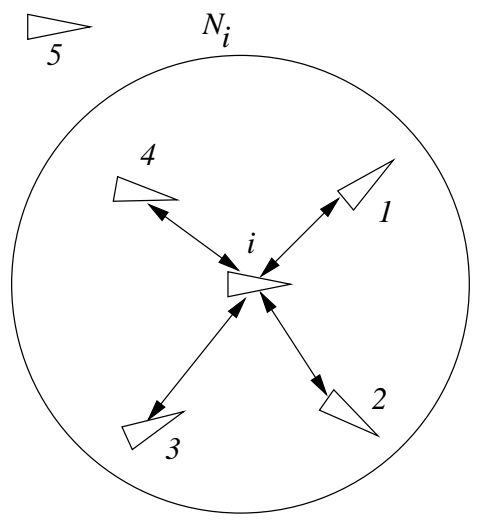

(a)

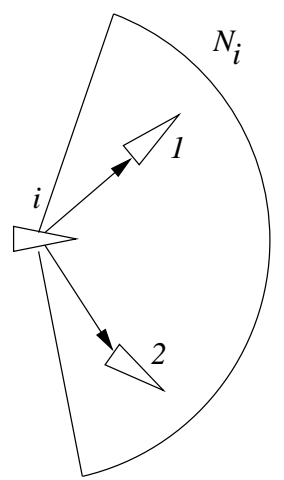

(b)

Figure 1: (a) A spherical neighborhood and (b) a conic neighborhood.

centered at $x=q_{i}$ in $\mathbb{R}^{d}$. Let $\mathcal{A}(q)=\left[a_{i j}(q)\right]$ be an adjacency matrix with 0,1 elements defined by

$$
a_{i j}(q)=\left\{\begin{array}{lr}
1, & q_{j} \in B\left(q_{i}, r\right), \\
0, & \text { otherwise }
\end{array}\right.
$$

for all $i, j \in \mathcal{I}$. The adjacency matrix $\mathcal{A}(q)$ defines a spatially induced graph $G(q)=(\mathcal{V}, \mathcal{E}(q))$ called a net. The set of neighbors of node $i$ in the net is defined as $N_{i}(q)=\left\{j: a_{i j}(q)>0\right\}$. The nets induced by spherical neighborhoods are undirected graphs, while the nets induced by conic neighborhoods are digraphs [10] as shown in Figure 1. A flock is a (weakly) connected net. In other words, each net consists of a number of flocks that are connected components of the net.

From equation (2), it is clear that the adjacency matrix induced by closed balls is nonsmooth. To define a smooth adjacency matrix, we redefine the adjacency elements as

$$
a_{i j}(q)=\rho\left(\left\|q_{j}-q_{i}\right\| / r\right)
$$

where $\rho: \mathbb{R}_{\geq 0} \rightarrow[0,1]$ is a smooth influence map $[10]$ given by

$$
\rho(z)=\left\{\begin{array}{lr}
1, & z \in[0, \delta] \\
\frac{1}{2^{k}}\left[1+\cos \left(\pi \frac{(z-\delta)}{(1-\delta)}\right)\right]^{k}, & z \in[\delta, 1] \\
0 & \text { otherwise. }
\end{array}\right.
$$

with parameters $\delta \in(0,1)$ and $k=1,2,3, \ldots$. One can show that $\rho(z)$ is a $C^{k}$-smooth function with the property that $\rho^{\prime}(z)=0$ over $[0, \delta) \cup(1, \infty)$ and $\left|\rho^{\prime}(z)\right|$ is uniformly upper-bounded in $z$. Let $a_{i j}(q)$ be the influence of node $i$ on node $j$. Then, node $i$ has no influence on node $j$ if $\left\|q_{j}-q_{i}\right\| \geq r$. This property is the primary reason that renders all the computational algorithms of this paper distributed. By definition, each node has an influence of 1 on itself.

Remark 1. For future use, without loss of generality, one can assume each node has no influence on itself. This assumption makes no difference in design and analysis of the cases of interest in this paper. 
A dynamic net is a net (or graph) with nodes that are dynamic systems. Each node has a state $x_{i}=\left(q_{i}, p_{i}\right)$, input $u_{i}$, and dynamics $\dot{x}_{i}=f\left(x_{i}, u_{i}\right)$ (given in (1)). Similar to mechanical systems, one can define potential and kinetic energies associated with dynamic nets. In the following, we provide a justification for our choice of the potential function.

Let us categorize a class of dynamic agents that attempt to maintain a fixed distance $d_{\alpha} \in(0, r)$ from other neighboring agents as $\alpha$-agents. To be more specific, consider the following set of algebraic distance-based constraints

$$
\left\|q_{j}-q_{i}\right\|=d_{\alpha}, \forall j \in N_{i}(q) \backslash\{i\}
$$

Any configuration $q=\left(q_{1}, \ldots, q_{n}\right) \in \mathbb{R}^{n d}$ satisfying (5) is called an $\alpha$-conformation. An $\alpha$ conformation is a realization of a structural $\alpha$-net that is a triplet $\left(n, d_{\alpha}, r\right)$. To quantify the degree in which the configuration of a fixed set of points is close to an $\alpha$-conformation, we construct a smooth and nonnegative potential function $V(q): \mathbb{R}^{n d} \rightarrow \mathbb{R}_{\geq 0}$. Let $\psi(z): \mathbb{R} \rightarrow \mathbb{R}_{\geq 0}$ be a smooth and nonnegative penalty function with a uniformly bounded differential. Then the following potential function

$$
V(q)=\sum_{i \neq j} a_{i j}(q) \psi\left(\left\|q_{j}-q_{i}\right\|-d_{\alpha}\right)
$$

is a nonnegative function that is smooth in all collision-free configurations (i.e. for all

$$
q \in Q_{\text {free }}:=\left\{q: q_{i} \neq q_{j}, \forall i, j \in \mathcal{I}\right\} .
$$

Moreover, the gradient $\nabla V(q)$ is globally uniformly bounded in $q$. The key property of $V(q)$ is that

$$
V(q)=0 \Longleftrightarrow q \text { is an } \alpha \text {-conformation. }
$$

Any configuration $q$ induces a net $G(q)$. We refer to the pair $(G(q), q)$ as a framenet. A framenet $(G(q), q)$ is called an $\alpha$-framenet if $q$ is an $\alpha$-conformation. The potential of all $\alpha$-framenets is zero. By "potential of a framenet", we mean potential of its configuration $q$. The potential $V(q)$ can be viewed as the "distance" between an arbitrary framenet $(G(q), q)$ and an $\alpha$-framenet which is a "desired framenet" satisfying the set of constraints in (5). Examples of $\alpha$-framenets with $n=4$ nodes are shown in Figure 2.

In the next section, we define a kinetic energy $K_{r}(p)$ that together with potential function $V(q)$ defines a structural energy (or Hamiltonian) for a dynamic net.

\section{$3 \quad$ Velocity Matching}

In this section, we discuss a consensus protocol that achieves velocity matching in a group of dynamic agents. The main velocity matching protocol used in this paper is a multi-dimensional and variable topology version of the agreement protocol (9) in [9]. Our approach for achieving velocity matching is to reduce a non-negative velocity mismatch (or disagreement) function to zero.

Define the operations Ave $(\cdot)$ and $\tilde{r}$ on vectors $x$ and $x_{i}$ as $\bar{x}=\operatorname{Ave}(x)=\left(\sum_{i=1}^{n} x_{i}\right) / n$ and $\tilde{x}_{i}=x_{i}-\bar{x}$, respectively. Following the idea of disagreement functions in multi-agent consensus 


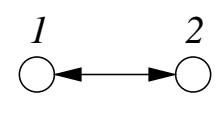

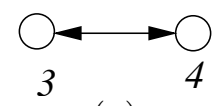

(a)

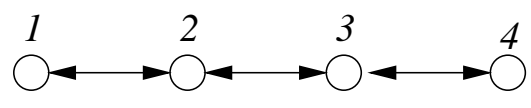

(c)

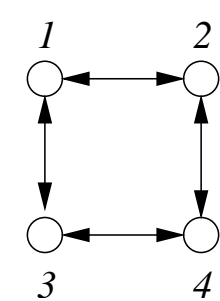

(b)

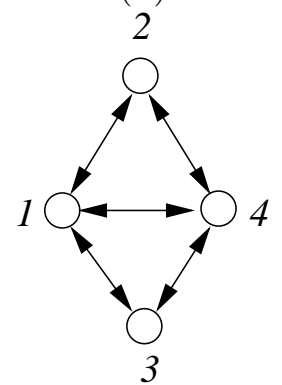

(d)

Figure 2: Four examples of $\alpha$-framenets.

problems $[9,7,8]$, we define the kinetic energy of the dynamic net as the following velocity mismatch function

$$
K_{r}(p)=\frac{1}{2} \sum_{i=1}^{n}\left\|\tilde{p}_{i}\right\|^{2} .
$$

The kinetic energy $K_{r}(p)$ does not depend on configuration $q$. The reason $K_{r}(p)$ is called a velocity mismatch function is that in case of zero velocity mismatch, or $K_{r}(p)=0$, we have $\tilde{p}_{i}=0$ for all $i \in \mathcal{I}$. This means that the velocities of all the agents are equal to $\bar{p}$ and thus match.

Note 1. Since, we assume $a_{i i}(q)=1$ for all $i$, it turns out that each node is its own neighbor, i.e. $i \in N_{i}(q)$ and therefore $\left|N_{i}(q)\right| \geq 1$. We use the notation $\hat{N}_{i}=N_{i} \backslash\{i\}$ to refer to the neighbors of node $i$ other than itself.

Here is the main use of the velocity mismatch function:

Proposition 1. (velocity matching protocol) Assume each dynamic agent in (1) applies the following velocity consensus protocol for dimension $d \geq 2$

$$
u_{i}^{v m}=c \sum_{j \in N_{i}(q)}\left(p_{j}-p_{i}\right), c>0
$$

In addition, suppose there exists a time $T>0$ such that $G(q(t))$ is a flock for all $t \geq T$. Then, the velocity of all agents asymptotically converge to Ave $(p(0))$ and match.

Proof. Let $u=u^{v m}$ (the superscript $v m$ stands for "velocity matching"). Since $\sum_{i} u_{i}=0$ for all $t, \operatorname{Ave}(p(t))$ is and invariant quantity and thus equal to $\bar{p}(0)=\operatorname{Ave}(p(0))$. Thus, $\dot{\tilde{p}}_{i}=u_{i}$ for all $i$. Let $\bar{A}(q)=\left[\bar{a}_{i j}(q)\right]$ be the adjacency matrix of the net $G(q)$ with 0,1 elements defined by 
$\bar{a}_{i j}(q)=\left\lceil a_{i j}(q)\right\rceil(\lceil\cdot\rceil$ denotes the ceiling function $)$. Define the $n d \times n d$ matrix $\hat{A}(q)=I_{d} \otimes \bar{A}(q)$ $(\otimes$ denotes the Kronecker product) and let $L(q)$ be the Laplacian matrix associated with the adjacency matrix $\hat{A}(q)$ (i.e. the diagonal matrix of the row sums of $\hat{A}(q)$ minus $\hat{A}(q)$ ). It turns out that $u=-c L(q) p$ where $L(q)$ is a symmetric Laplacian matrix for all $q$ satisfying $L(q) p=L(q) \tilde{p}$ and the following sum of squares (SOS) property holds

$$
p^{T} L(q) p=\frac{1}{2} \sum_{i j \in \mathcal{E}(q)}\left\|p_{j}-p_{i}\right\|^{2}
$$

where $\|\cdot\|$ denotes the 2 -norm in $\mathbb{R}^{d}$. Given the velocity consensus (or matching) protocol, we have

$$
\dot{K}_{r}=\tilde{p}^{T} u=-c \tilde{p}^{T} L(q) \tilde{p} \leq 0, \forall t \geq 0
$$

which means the velocity mismatch dynamics

$$
\dot{\tilde{p}}=-c L(q) \tilde{p}
$$

is stable. Due to the assumption that $G(q(t))$ is a connected graph for all $t>T, L(q(t))$ has rank $n-1$ for all $t>T$ and

$$
\dot{K}_{r}=-c \tilde{p}^{T} L(q) \tilde{p}<0, \forall \tilde{p} \neq 0
$$

Thus, $K_{r}(p)$ is a valid Lyapunov function for the velocity mismatch dynamics for all $t>T$ and $\tilde{p}$ globally asymptotically converges t o zero. In other words, the velocity of all agents asymptotically match.

Remark 2. In the proof of Proposition 1, notice that $\tilde{p}^{T} L(q) \tilde{p}=0$ implies $\tilde{p}_{i}=\tilde{p}_{j}$ for all the edges $i j \in \mathcal{E}(q)$. If $G(q)$ is connected, then all the $\tilde{p}_{i}$ 's are equal. But $\sum_{i} \tilde{p}_{i}=0$, thus $\tilde{p}=0$. This argument proves that for a connected graph $\tilde{p}^{T} L(q) \tilde{p}>0$ for all $\tilde{p} \neq 0$.

Remark 3. Given the velocity matching protocol in Proposition 1, one can show that $\|p(t)\|$ remains uniformly bounded for all $t \geq 0$. This follows from the fact that

$$
\frac{d}{d t} \mid\|p(t)\|^{2}=p^{T} u^{v m}=-p^{T} L(q) p \leq 0 .
$$

Thus, $\|p(t)\| \leq\|p(0)\|$, for all $t \geq 0$.

Remark 4 . The velocity matching protocol in (9) can be replaced with

$$
u_{i}^{v m}=c \sum_{j \in N_{i}(q)} a_{i j}(q)\left(p_{j}-p_{i}\right), c>0
$$

with $u_{i}^{v m}=0$ for $N_{i}(q)=\emptyset$ and the result of Proposition 1 would still hold. The proof follows the same line of argument with a minor difference that $L(q)$ is the Laplacian matrix of the weighted adjacency matrix $I_{d} \otimes \mathcal{A}(q)$ and the following weighted SOS property holds:

$$
p^{T} L(q) p=\frac{1}{2} \sum_{i j \in \mathcal{E}(q)} a_{i j}(q)\left\|p_{j}-p_{i}\right\|^{2}
$$


According to the velocity matching protocol in (9), we have

$$
u_{i}^{v m}=c\left|N_{i}(q)\right|\left(\frac{\sum_{j \in N_{i}(q)} p_{j}}{\left|N_{i}(q)\right|}-p_{i}\right)=k(q)\left(p_{i}^{a v}-p_{i}\right)
$$

with $k(q)=c\left|N_{i}(q)\right| \geq c>0$ and $p_{i}^{a v}=\sum_{j \in N_{i}(q)} p_{j} /\left|N_{i}(q)\right|$. Therefore, the velocity of each agent changes towards the average velocity of its neighbors. For the special case of a node that has no neighbors other than itself, or an isolated node with $N_{i}(q)=\{i\}$, we get $k(q)=c>0$ and $p_{i}^{a v}=p_{i}$. As a result, $u_{i}^{v m}=0$ and an isolated agent moves along a straight line.

In [12], an alignment version of the rule in (17) was studied where all the velocities are replaces by heading angles of the agents. This rule alone has to do nothing with flocking. However, if the density of the agents in a restricted area is relatively high, the net becomes connected and all the agents asymptotically match their velocities and therefore move towards the same direction. This group behavior might be perceived as flocking by some researchers [12]. Here, we do not consider such a group behavior as flocking where no configuration-dependent constraints are desired to be satisfied by the agents.

An interesting phenomenon occurs whenever there is low density of agents in a restricted area of initial positions. This leads to disconnectivity of the net $G(q(t))$ at time $t=0$ and (perhaps) future times. Such disconnectivity of the net creates smaller flocks of agents or "Islands of Agents". In each island, all the agents asymptotically move towards the same direction. However, the islands as a whole incohesively disperse in different directions depending on the average values of the initial velocities of agents in each flock. Apparently, some of these flocks might merge upon inter-flock collisions and form larger flocks. Two flocks collide whenever one agent from one flock becomes the neighbor of an agent in the other flock.

\section{The $(\alpha, \alpha)$ Flocking Protocol and Reynolds Rules}

Consider the following structural energy of a dynamic net

$$
H_{s}(q, p)=V(q)+K_{r}(p)
$$

where

$$
V(q)=\sum_{i=1}^{n} \sum_{j \in N_{i}(q), j \neq i} a_{i j}(q) \psi_{\alpha}\left(\left\|q_{j}-q_{i}\right\|-d_{\alpha}\right)
$$

and the penalty function $\psi_{\alpha}(z)$ is a scalar function in the form

$$
\psi_{\alpha}(z)=\frac{a+b}{2}\left[\sqrt{1+(z+c)^{2}}-\sqrt{1+c^{2}}\right]+\left(\frac{a-b}{2}\right) z
$$

with uniformly bounded derivative and parameters $b>a>0$ and $c=(b-a) / 2 \sqrt{a b}$.

Remark 5. This choice of $\psi_{\alpha}(z)$ guarantees that $\sigma_{\alpha}(z)=\psi_{\alpha}^{\prime}(z)$ given by

$$
\sigma_{\alpha}(z)=\frac{a+b}{2} \frac{z+c}{\sqrt{1+(z+c)^{2}}}+\frac{a-b}{2}
$$

is an asymmetric sigmoidal function satisfying the properties $\sigma_{\alpha}(0)=0, z \sigma_{\alpha}(z)>0, \forall z \neq 0$, $\lim _{z \rightarrow+\infty} \sigma_{\alpha}(z)=a$, and $\lim _{z \rightarrow-\infty} \sigma_{\alpha}(z)=-b$. As a result, the potential $\psi_{\alpha}\left(\left\|q_{j}-q_{i}\right\|-d_{\alpha}\right)$ between two neighboring agents is more repulsive than attractive because of $a<b$. 
Remark 6. The use of inter-agent potentials that are more repulsive than attractive is crucial in avoiding a negative phenomenon that creates "Dense Islands of Agents" where the agents remain tightly close to each other in each island (i.e, small flock).

According to [10], given a state feedback $u=k(q, p)$, flocking is achieved for a dynamic net with structural energy $H_{s}(q, p)$ if the following conditions hold:

i) the structural energy $H_{s}(q(t), p(t))$ is rendered monotonically decreasing, i.e. $\dot{H}_{s} \leq 0$,

ii) the structural energy $H_{s}(q(t), p(t))$ asymptotically vanishes,

iii) the net $G(q(t))$ asymptotically becomes a flock.

In $[6,10]$, dissipation of structural energy is proposed as an approach to achieve flocking. Furthermore, the following distributed state feedback

$$
u_{i}=\sum_{j \in \hat{N}_{i}(q)} \phi_{\alpha}\left(\left\|q_{j}-q_{i}\right\|-d_{\alpha}\right) \mathbf{n}_{i j}+c_{d}\left(p_{j}-p_{i}\right), c_{d}>0
$$

called the $(\alpha, \alpha)$ protocol for a detailed analysis) was used to render the system dissipative (see [6] for a detailed analysis). Here, $\hat{N}_{i}$ is the set of neighbors of agent $i$ excluding itself, $c_{d}>0$ is a dissipation coefficient, $\mathbf{n}_{i j}$ is the unit vector connecting $q_{i}$ to $q_{j} \neq q_{i}$, and $\phi_{\alpha}: \mathbb{R} \rightarrow \mathbb{R}$ is an action function (i.e. the derivative of a penalty function) defined by

$$
\phi_{\alpha}(z)=\frac{d \hat{\psi}_{\alpha}(z)}{d z}, \hat{\psi}_{\alpha}(z)=\rho\left(\frac{z+d_{\alpha}}{r}\right) \psi_{\alpha}(z)
$$

Apparently, the first term in (22) is a gradient-based term due to the objective of an $\alpha$-agent. We refer to this term as the motion planning term and denote it by

$$
u_{i}^{m p}=\sum_{j \in \hat{N}_{i}(q)} \phi_{\alpha}\left(\left\|q_{j}-q_{i}\right\|-d_{\alpha}\right) \mathbf{n}_{i j}
$$

The $(\alpha, \alpha)$ protocol can be expressed as the sum of a motion planning term and a velocity matching term, i.e.

$$
u_{i}=u_{i}^{m p}+u_{i}^{v m}, \quad \forall i \in \mathcal{I} .
$$

In the following, we demonstrate that the first and second rules of Reynolds can be interpreted as special cases of the motion planning term of the $(\alpha, \alpha)$ protocol in $(22)$.

Let us define a set of weights associated with the edges of a net called the stress weights [10] as

$$
s_{i j}(q)=\frac{\phi_{\alpha}\left(\left\|q_{j}-q_{i}\right\|-d_{\alpha}\right)}{\left\|q_{j}-q_{i}\right\|}, \quad q_{j} \neq q_{i}, j \in N_{i}(q) \backslash\{i\}
$$

and $s_{i j}(q)=0$, otherwise. Using this notation, the motion planning term of the $(\alpha, \alpha)$ flocking protocol can be written as

$$
u_{i}^{m p}=\sum_{j \in N_{i}(q)} s_{i j}(q)\left(q_{j}-q_{i}\right)
$$

which allows one to interpreter the $(\alpha, \alpha)$ protocol as a distributed PD Controller with nonlinear state-dependent gains. 
The net $G(q)$ with adjacency elements $s_{i j}(q)$ can be viewed as a spatially induced weighted graph with sign indefinite weights. The Laplacian of such graphs are no longer positive semidefinite. Let $S(q)=\left[s_{i j}(q)\right]$ be the stress matrix of the net $G(q)$. We define three new sets of neighbors called friends for an agent. The friends of an agent are determined by the stress matrix. Define the set of positive, negative and neutral friends of agent $i$ as

$$
\begin{aligned}
F_{i}^{+} & =\left\{j \in N_{i}(q): s_{i j}(q)>0\right\}, \\
F_{i}^{-} & =\left\{j \in N_{i}(q): s_{i j}(q)<0\right\}, \\
F_{i}^{0} & =\left\{j \in N_{i}(q): s_{i j}(q)=0\right\}
\end{aligned}
$$

respectively. We have $N_{i}(q)=F_{i}^{+} \cup F_{i}^{-} \cup F_{i}^{0}$ and $i \in F_{i}^{0}$, i.e. each agent is a neutral friend of itself. Define the local stress of agent $i$ as the sum

$$
S_{i}(q)=\sum_{j \in N_{i}(q)} s_{i j}(q)=S_{i}^{+}(q)+S_{i}^{-}(q) .
$$

with $S_{i}^{ \pm}(q)=\sum_{j \in F_{i}^{ \pm}} s_{i j}(q)$. Notice that by definition $S_{i}^{+}(q)>0$ and $S_{i}^{-}(q)<0$. The following two cases arise:

Case i) $S_{i}(q) \neq 0$ : In case of a nonzero local stress for agent $i$, we can define the center of neighbors $(C O N)$ of agent $i$ as the following weighted sum of the positions of its neighbors other than itself

$$
q_{i}^{a v}=\frac{1}{S_{i}(q)} \sum_{j \in N_{i}(q)} s_{i j}(q) q_{j}
$$

and the motion planning term can be rewritten as

$$
u_{i}^{m p}=S_{i}(q)\left(q_{i}^{a v}-q_{i}\right)
$$

which means agent $i$ moves towards/away from the center of its neighbors depending on the sign of its nonzero local stress. The last expression in italic that is the description of the motion planning term in (33) encompasses both the "Flock Centering" rule and the "Collision Avoidance" rule of Reynolds.

The geometric interpretation of the motion planning term in (33) can be stated as follows. Define the center of positive and negative friends of agent $i$ as follows

$$
\begin{aligned}
q_{i}^{a v+} & =\frac{1}{S_{i}^{+}(q)} \sum_{j \in F_{i}^{+}(q)} s_{i j}(q) q_{j}, \\
q_{i}^{a v-} & =\frac{1}{S_{i}^{-}(q)} \sum_{j \in F_{i}^{-}(q)} s_{i j}(q) q_{j} .
\end{aligned}
$$

Then

$$
q_{i}^{a v}=\lambda_{1} q_{i}^{a v+}+\lambda_{2} q_{i}^{a v-},
$$

with $\lambda_{1,2}:=S_{i}^{ \pm}(q) / S_{i}(q)$ satisfying $\lambda_{1}+\lambda_{2}=1$, i.e. agent $i$ with nonzero local stress applies an input (or moves) towards/away from the direction of the line connecting $q_{i}$ to a point 


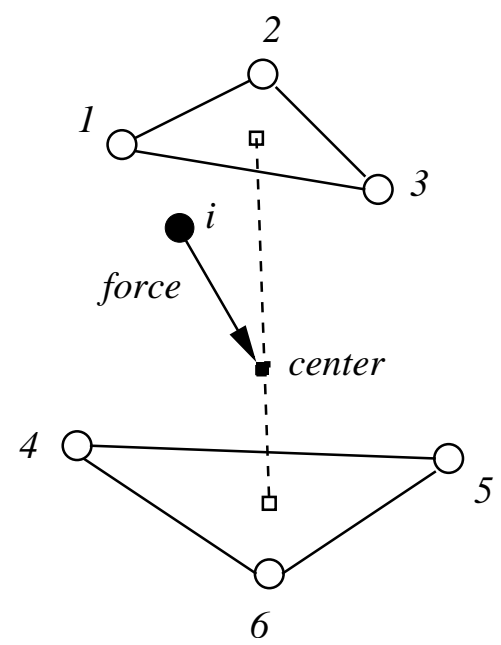

(a)

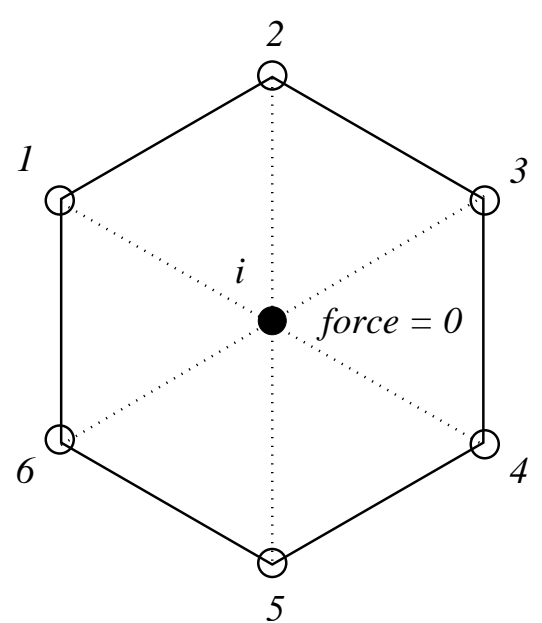

(b)

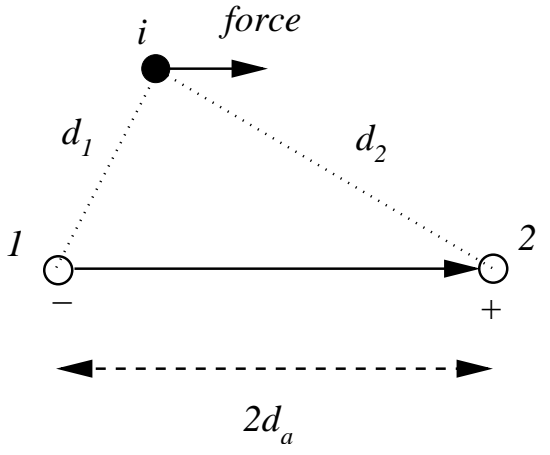

(c)

Figure 3: The action of agent $i$ under different local stresses: (a) $S_{i}(q) \neq 0$, (b) $S_{i}(q)=0$ and $u_{i}^{m p}=0$, and (c) $S_{i}(q)=0$ and $u_{i}^{m p} \neq 0$.

in the convex hull of the center of positive friends of $i$ and the center of negative friends of $i$ depending on whether $\lambda_{1}>0$ or $\lambda_{2}>0$. This rule is schematically demonstrated in Figure 3(a). Node $i=7$ has three negative and three positive friends. A force along the vector connecting $q_{i}$ to $q_{i}^{a v}$ (marked by a filled square) is applied to agent $i$. The centers of positive and negative friends are marked by unfilled squares. To show that the scenario in Figure 3(a) is realistic, let $d_{j}$ denote the distance between agent $i$ and agent $j$ for $j=1, \ldots, 6$ and assume $d_{j}<d_{j+1}$. In addition, suppose $d_{\alpha}$ satisfies $d_{3}<d_{\alpha}<d_{4}$. Then, the sets of signed friends of agent $i$ are determined as follows: $F_{i}^{-}=\{1,2,3\}, F_{i}^{+}=\{4,5,6\}$, and $F_{i}^{0}=\{i\}$.

Case ii) $S_{i}(q)=0$ : In this case, the local stress of agent $i$ is zero and therefore $S_{i}^{+}(q)=-S_{i}^{-}(q)$. If agent $i$ has no positive (or negative) friends, then $u_{i}^{m p}=0$ and agent $i$ with $u_{i}=u_{i}^{m p}$ does not change its velocity (Remark: in reality due to velocity matching term, agent $i$ still might change its velocity). An example of this scenario is shown in Figure 3(b) with the condition that each edge of the hexagon is of length $d_{\alpha}$.

Now, suppose agent $i$ at least has one positive friend. Then, due to $S_{i}^{+}(q)=-S_{i}^{-}(q)>0$, it should at least have one negative friend as well. Assume $F_{i}^{+}(q) \neq \emptyset$, we get

$$
\begin{aligned}
u_{i}^{m p} & =\sum_{j \in N_{i}(q)} s_{i j}(q) q_{j}-S_{i}(q) q_{i} \\
& =\sum_{j \in F_{i}^{+}} s_{i j}(q) q_{j}+\sum_{j \in F_{i}^{-}} s_{i j}(q) q_{j} \\
& =S_{i}^{+}(q) q_{i}^{a v+}+S_{i}^{-}(q) q_{i}^{a v-}
\end{aligned}
$$


which due to $S_{i}^{+}(q)=-S_{i}^{-}(q)>0$ can be expressed as

$$
u_{i}^{m p}=S_{i}^{+}(q)\left(q_{i}^{a v+}-q_{i}^{a v-}\right) .
$$

Equation (37) can be translated as follows: agent $i$ applies an input (or moves) along the direction of the vector connecting the center of negative friends of $i$ to the center of positive friends of $i$. Note that $q_{i}^{a v \pm}$ is inside the convex hull of the positions of the positive/negative friends of agent $i$.

This scenario in case ii) is schematically shown in Figure 3(c) for agent $i$ with one positive friend and one negative friend. Assume that the distance between these two friends is $2 d_{\alpha}$. Assuming that each edge of the hexagon in Figure 3(c) is of length $d_{\alpha}$, the set of singed friends of agent $i$ can be specified as $F_{i}^{-}=\{1\}, F_{i}^{+}=\{2\}$, and $F_{i}^{0}=\{i\}$. Since there is only a single positive (negative) friend, the center of positive (negative) friends is the position of the positive (negative) friend. In Figure 3(c), The motion planning term $u_{i}^{m p}$ is aligned with the vector connecting these two signed friends.

Remark 7. In Figure 3(c), let $d_{1}$ and $d_{2}$ denote the distance between $q_{i}$ and agents 1 and 2 . The set of points $q_{i}$ in $\mathbb{R}^{2}$ with zero local stress satisfy

$$
\frac{\phi_{\alpha}\left(d_{1}-d_{\alpha}\right)}{d_{1}}+\frac{\phi_{\alpha}\left(d_{2}-d_{\alpha}\right)}{d_{2}}=0
$$

Apparently, $d_{1}=d_{2}=d_{\alpha}$ is a trivial solution of this equation.

If the velocity of agent $i$ is equal to all of its neighbors, then $u_{i}^{v m}=0$ and $u_{i}=u_{i}^{m p}$ which encompasses the first two rules of Reynolds. On the other hand, if all the friends of agent $i$ are neutral friends, then $u_{i}^{m p}=0$. Thus, in this case, $u_{i}=u_{i}^{v m}$. A relative equilibrium is reached whenever all the friends of agent $i$ are neutral friends and the velocity of $i$ matches the velocity of all of its neighbors.

We have established that the $(\alpha, \alpha)$ protocol encompasses more sophisticated versions of all three flocking rules of Reynolds introduced in [11]. The $(\alpha, \alpha)$ protocol is only valid for flocking in lack of any obstacles. For flocking with obstacle avoidance, two other types of agents called $\beta$ and $\gamma$ agents are introduced in [6].

Remark 8. The "Obstacle Avoidance" rule of Reynolds has to do nothing with avoiding collision to environmental obstacles. Instead it has to do with collision between an agent and its nearby flockmates. The motion planning term performs this agent-to-agent collision avoidance in a practical sense. By "practical", we mean each agent makes an "attempt" to avoid collision to other nearby flockmates. This attempt might or might not be successful to avoid a collision. Therefore, achieving practical agent-to-agent collision avoidance does not mean that no collisions would occur among the agents.

The scenario in case ii) is new and has not been foreseen in the work of Reynolds [11]. In fact, no equations were presented by Reynolds that specify the flocking rules. Therefore, it is hard to imagine that the advanced versions of each rule of Reynolds with state-dependent weights could be predicted from the work in [11]. The justification presented in [11] for "Flock Centering" was that all agents try to stay near the center (i.e. average position) of the flock members. Since no agent knows the center of the overall flock, "each agent moves towards the center of all of its 
neighbors". It is obvious that the derivation of the motion planning term of the $(\alpha, \alpha)$ protocol has to do nothing with simple intuitive reasonings by Reynolds [11]. The weights used to calculate this "center of neighbors" are not arbitrary or constant in our work. Here, the weights are provided in explicit form and the convergence (or stability) analysis presented in [6] justifies the proposed $(\alpha, \alpha)$ flocking protocol.

The following result shows that the $(\alpha, \alpha)$ protocol for the overall flock is a PD controller with matrix-gains that are state-dependent graph Laplacians.

Proposition 2. The $(\alpha, \alpha)$ protocol for the flock as a whole can be expressed in the following form

$$
u=-L_{s}(q) q-c_{d} L(q) p=-L_{s}(q) \tilde{q}-c_{d} L(q) \tilde{p}, c_{d}>0
$$

where $L_{s}(q)$ and $L(q)$ are the Laplacian matrices associated with the adjacency matrices $I_{d} \otimes S(q)$ and $I_{d} \otimes \bar{A}(q)$.

Proof. The proof follows from the fact that the motion planning term and the velocity matching term of the $(\alpha, \alpha)$ protocol have identical forms given by

$$
f_{i}=\sum_{j \in N_{i}} w_{i j}\left(x_{j}-x_{i}\right), \quad x_{i} \in \mathbb{R}^{d}, \forall i
$$

with the difference that $w_{i j}=s_{i j}(q)$ and $x_{i}=q_{i}$ for the motion planning term and $w_{i j}=\bar{a}_{i j}(q)=$ $\left\lceil a_{i j}(q)\right\rceil$ and $x_{i}=p_{i}$ for the velocity matching term. The Kronecker product of the $d \times d$ identity matrix with the adjacency matrix is necessary to create a Laplacian matrix with appropriate dimensions.

\section{Simulation Results: Flocking Towards a Sink}

Consider a group of $n=100 \alpha$-agents starting from random initial positions with zero initial velocities. The desired task is to perform flocking in presence of a sink at $\left(q_{d}, p_{d}\right)=(0,0)$ and in lack of any environmental obstacles. According to [6], the following translational PD controller

$$
f_{i}=-c_{1} q_{i}-c_{2} p_{i}, \quad i \in \mathcal{I}, c_{1}, c_{2}>0
$$

has to be added to the control from the $(\alpha, \alpha)$ protocol. The $\alpha$-agents need to maintain an interagent distance equal to $d_{\alpha}=7$ with $r=1.2 d_{\alpha}$. Figure 4 shows an initially disconnected net that evolves into a flock after going through a finite number of switching events that add more edges to the initial net. The topology of the flock is asymptotically invariant. One of our future objectives is to prove that the combination of the $(\alpha, \alpha)$ protocol and the translational controller in (40) leads to automatic assembly of connected mobile networks of agents.

\section{Conclusion}

This work relies on a dynamic graph theoretic framework for flocking developed by the author in $[6,10]$. The primary flocking protocol that is used for flocking in lack of environmental obstacles is called the $(\alpha, \alpha)$ protocol [6,10]. This protocol describes the behavior of an $\alpha$-agent in its interactions with other neighboring $\alpha$-agents. 
In this paper, we demonstrated that the $(\alpha, \alpha)$ protocol encompasses all three flocking rules of Reynolds as special cases. A geometric interpretation of the motion planning term of the $(\alpha, \alpha)$ protocol was given.

We showed that the $(\alpha, \alpha)$ protocol for the flock as a whole is a PD controller with statedependent matrix-gains that are graph Laplacians. These Laplacian matrices are determined by the stress matrix and the upper-truncated adjacency matrix of the net. Moreover, two negative phenomena in flocking were discussed. Specifically, creation of disconnected and dense islands of agents. Both of these phenomena lead to creation of multiple flocks that incohesively disperse in different directions. Simulation results are provided that demonstrate flocking for 100 agents in presence of a sink.

\section{Acknowledgments}

This research is supported in part by AFOSR under the grant F49620-01-1-0361 and by DARPA under the grant F33615-98-C-3613. Many thanks goes to Richard Murray for discussing the problem of performing split/rejoin maneuvers with the author a few years ago. The author also would like to thank Bryan Stout for his comments regarding the work in [6].

\section{References}

[1] D. E. Chang, S. Shadden, J. Marsden, and R. Olfati Saber. Collision Avoidance for Multiple Agent Systems. Proc. of the IEEE Conf. on Decision and Control, February 2003.

[2] V. Gazi and K. Passino. Stability analysis of swarms. IEEE Trans. on Automatic Control (to appear), May 2003.

[3] D. Helbing, I. Farkas, and T. Vicsek. Simulating dynamical features of escape panic. Nature, 407:487-490, 2000 .

[4] A. Jadbabaie, J. Lin, and S. A. Morse. Coordination of groups of mobile agents using nearest neighbor rules. To appear in the IEEE Trans. on Automatic Control, 2002.

[5] Y. Liu, K. Passino, and M. M. Polycarpou. Stability analysis of $M$-dimensional asynchronous swarms with a fixed communication topology. IEEE Trans. On Automatic Control, 48(1):7695, January 2003.

[6] R. Olfati Saber. Flocking with Obstacle Avoidance. Technical Report 2003-006, California Institute of Technology, Control and Dynamical Systems, Pasadena, California, February 2003, http://resolver.caltech.edu/CaltechCDSTR: 2003.006.

[7] R. Olfati Saber and R. M. Murray. Agreement Problems in Networks with Directed Graphs and Switching Topology. Proc. of the IEEE Conference on Decision and Control, December 2003.

[8] R. Olfati Saber and R. M. Murray. Consensus problems in networks of agents with switching topology and time-delays. Submitted to the IEEE Trans. on Automatic Control, April 2003. 
[9] R. Olfati Saber and R. M. Murray. Consensus Protocols for Networks of Dynamic Agents. Proc. of the American Control Conference, June 2003.

[10] R. Olfati Saber and R. M. Murray. Flocking with Obstacle Avoidance: Cooperation with Limited Communication in Mobile Networks. Proc. of the IEEE Conference on Decision and Control, December 2003.

[11] C. W. Reynolds. Flocks, herds, and schools: a distributed behavioral model. Computer Graphics (ACM SIGGRAPH '87 Conference Proceedings), 21(4):25-34, July 1987.

[12] T. Vicsek, A. Cziroók, E. Ben-Jacob, and O. Cohen, I. Shochet. Novel type of phase transition in a system of self-deriven particles. Physical Review Letters, 75(6):1226-1229, August, 1995. 

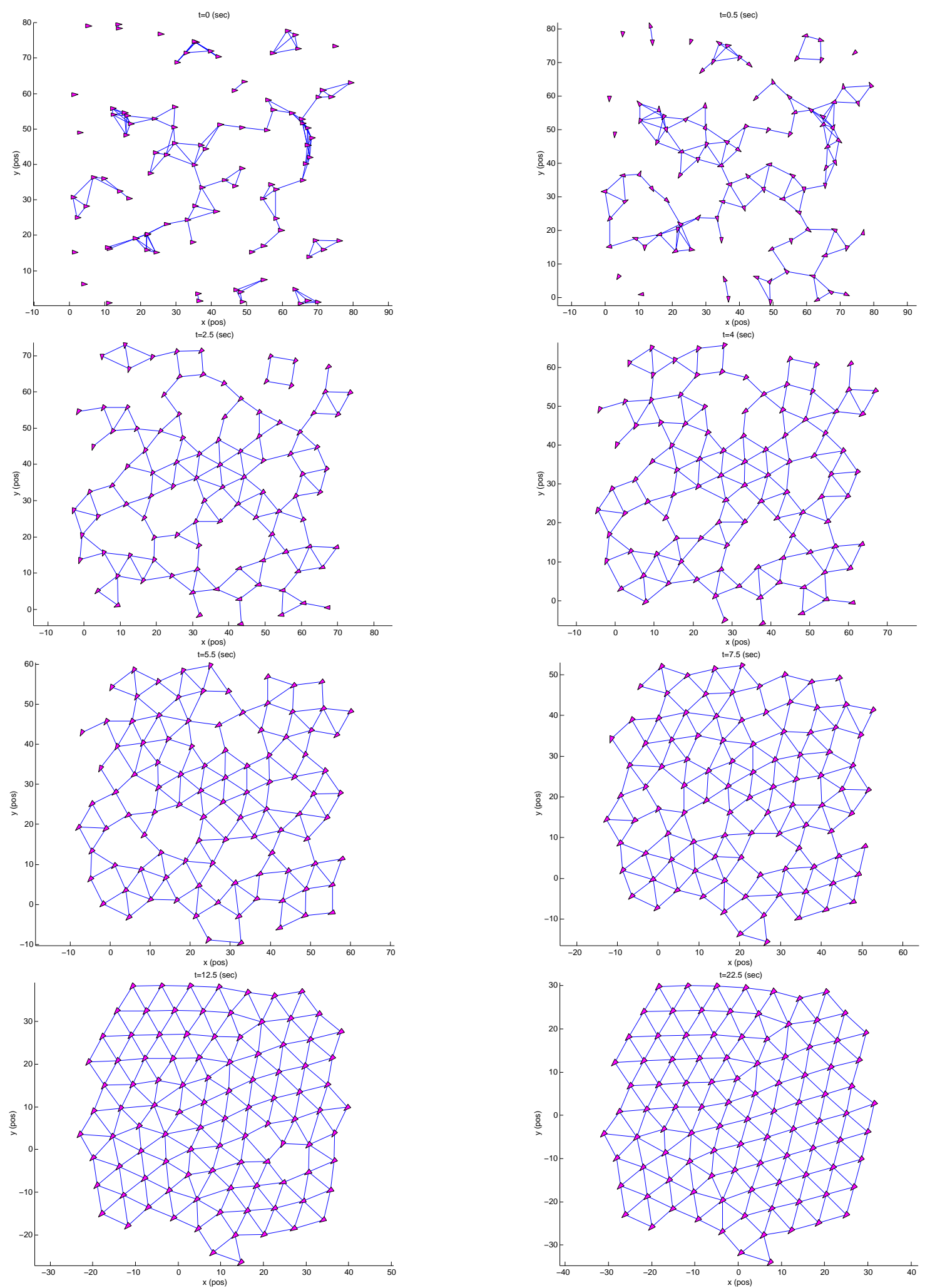

Figure 4: Consecutive snapshots of conformation of a flock from a net via flocking of $n=100$ agents in presence of a sink at $(0,0)$. 